\title{
Optical properties of the lens: An explanation for the zones of discontinuity
}

\author{
Mehdi Bahrami a , Masato Hoshino ${ }^{b}$, Barbara Pierscionek ${ }^{a,}{ }^{*}$, Naoto Yagi ${ }^{b}$, Justyn Regini ${ }^{c}$, \\ Kentaro Uesugi ${ }^{\text {b }}$ \\ a Faculty of Science, Engineering and Computing, Kingston University London, Kingston-upon-Thames, KT1 2EE, United Kingdom \\ b Japan Synchrotron Radiation Research Institute (SPring8), Sayo, Hyogo, Japan \\ c School of Optometry and Vision Sciences, Cardiff University, Cardiff, United Kingdom
}

\section{A R T I C L E I N F O}

\section{Article history:}

Received 28 March 2014

Accepted in revised form 13 May 2014

Available online 29 May 2014

\section{Keywords:}

eye lens

refractive index

zones of discontinuity

light scatter

implant design

\begin{abstract}
A B S T R A C T
The structural basis of zones of discontinuity in the living human eye lens has not been elucidated, and there is no conclusive explanation for what relevance they may have to the structure and function of the lens. Newly developed synchrotron radiation based X-ray Talbot interferometry has enabled the detection of subtle fluctuations in the human eye lens which, when used in mathematical modelling to simulate reflected and scattered light, can recreate the image of the lens seen in the living human eye. The results of this study show that the zones of discontinuity may be caused by subtle fluctuations in the refractive index gradient as well as from random scattering in the central regions. As the refractive index contours are created by cell layers with progressively varying protein concentrations, the zones are linked to growth and will contain information about ageing and development. The index gradient is important for image quality and fluctuations in this gradient may add to quality optimisation and serve as models for designs of new generation implant lenses.
\end{abstract}

(C) 2014 The Authors. Published by Elsevier Ltd. This is an open access article under the CC BY-NC-ND license (http://creativecommons.org/licenses/by-nc-nd/3.0/).

\section{Introduction}

The growth of the lens, by accrual of new fibre cells over existing tissue with no concomitant tissue losses, creates a concentric structure of layers varying in age and in protein content. When observed in vivo, using slit lamp biomicroscopy, distinct regions are seen in the lens and these have been labelled to accord with the certain stages of life at which the tissue within any given region was synthesised (Vogt, 1919, 1931, Mann, 1925; Goldmann, 1937). Their presence is a manifestation of growth processes and collectively, the regions have been called zones of discontinuity (Vogt, 1931). They have been considered to manifest sharp demarcations in the refractive index (Mann, 1925; Huggert, 1948) or to indicate scatter (Yaroslavsky et al., 1994). Subsequent studies have found that the refractive index does not alter as abruptly as previously suggested (Mann, 1925; Huggert, 1948) but increases relatively gradually from periphery to centre (reviewed in Pierscionek and Regini, 2012).

A biomicroscopic image of the lens seen in the living eye is formed by light that is back scattered and reflected from internal

\footnotetext{
* Corresponding author. Tel.: +44 2084174821.

E-mail address: b.pierscionek@kingston.ac.uk (B. Pierscionek).
}

structures. In a healthy eye, the light scatter that contributes to the zones of discontinuity does not impair vision. Any further interpretation of the biological characteristics of the zones of discontinuity requires a better understanding of the structural features which form the scattering sources and the optical characteristics of the lens.

The lens has a gradient refractive index and the proportion and direction of scattered light depends on the steepness of the refractive index gradient as well as on how smoothly varying it is (Born and Wolf, 1970). Recently, small fluctuations or kinks in the refractive index gradient of animal lenses have been found along the optic axis and in the equatorial plane (Hoshino et al., 2011) and it has been hypothesised that these fluctuations may account, at least in part, for the zones of discontinuity (Pierscionek and Regini, 2012).

Mathematical models can be used to reconstruct the refractive index gradient and fluctuations within it as seen in the axial region and to extend these paraxially, adhering to the concentric lamellar structure of the lens that gives rise to iso-indicial contours. Measurements taken along the optic axes of human lenses using X-ray synchrotron radiation were used to model and simulate biomicroscopic images. The findings suggest a direct relationship between zones of discontinuity and the refractive index of the lens. 


\section{Methods}

\subsection{X-ray Talbot interferometry}

Human eyeball samples (16, 35 and 48 years old), from which corneal discs were removed for transplantation, were obtained from the Bristol Eye Bank (UK) and transported frozen to the SPring-8 synchrotron radiation facility in Japan. Ethical approval was granted by the National Health Service (NHS) committee (Oxford, UK). Samples were thawed within $24 \mathrm{~h}$ of measurement and lenses removed from eyeballs and set in a physiologicallybalanced agarose gel within a specially designed sample holder. Refractive index of lenses was measured using the X-ray Talbot grating interferometer (Momose, 2005; Momose et al., 2003) as described in Hoshino et al. (2011) constructed at the bending magnet beamline BL20B2 at Spring-8. Briefly, the instrument utilises a monochromatic X-ray beam, tuned to $25 \mathrm{keV}$ that passes through a $\mathrm{Si}(111)$ double crystal monochromator and two transmission gratings: a phase grating (G1) made of tantalum and an absorption grating (G2) made of gold with pattern thicknesses $2.1 \mu \mathrm{m}$ and $16.6 \mu \mathrm{m}$ respectively and pitch of $10 \mu \mathrm{m}$. Moiré fringes generated by G1 and G2 were detected by the beam monitor and a scientific CMOS detector (ORCA Flash 4.0. Hamamatsu Photonics). For phase retrieval, G2 was shifted with a Piezo stage and 5-step 'on-the-fly' fringe-scan method used. The measurement time per sample took $82 \mathrm{~min}$ and around 900 scans were made for each lens.

\subsection{Modelling geometric scatter}

Geometric scattering in a discrete medium can be seen as reflections at interfaces where there is a change in the refractive index. The intensity of this reflection is described by the Fresnel reflection equation:

$$
\begin{aligned}
R=\frac{1}{2}\left(\left|\frac{n 1 \cos \theta-n 2 \sqrt{1-\left(\frac{n 1}{n 2} \sin \theta\right)^{2}}}{n 1 \cos \theta+n 2 \sqrt{1-\left(\frac{n 1}{n 2} \sin \theta\right)^{2}}}\right|^{2}\right. \\
+\left|\frac{n 1 \sqrt{1-\left(\frac{n 1}{n 2} \sin \theta\right)^{2}}-n 2 \cos \theta}{n 1 \sqrt{1-\left(\frac{n 1}{n 2} \sin \theta\right)^{2}}+n 2 \cos \theta}\right|
\end{aligned}
$$

where $R$ is the reflection coefficient, $\theta$ is the angle between the incident ray and the normal vector of the surface, and $n 1$ and $n 2$ are the refractive indices of two neighbouring points.

In a gradient refractive index (GRIN) medium, $n 1$ and $n 2$ can be described as $n(x 1, y 1, z 1)=n 1$ and $n(x 2, y 2, z 2)=n 2$, where $n(x, y, z)$ is the refractive index function of the medium at each point $(x, y, z)$. If $(x 2, y 2, z 2)=(x 1, y 1, z 1)+\vec{\Delta}$, where $|\vec{\Delta}|$ approaches 0 , the incremental difference between $n 1$ and $n 2$ can closely approximate a continuous function. The gradient of the refractive index function $n(x, y, z)$ can therefore be simulated by very small increments and equation (1) applied iteratively for each sequential increment in refractive index along the gradient. Equation (1) was applied to spherical lenses with different radially varying refractive index profiles: linear, quadratic, cubic and quartic to determine the pattern of reflected light and to see how these vary with different refractive index forms.

Equation (1) was also applied to experimentally measured refractive index gradients in three human lenses, the surface shapes of which were extracted from the X-ray images. The number of iterative steps varied depending on lens size and ranged between 550 and 700 for on axis calculations; for calculations in 2-dimensions this ranged from $6.05 \times 10^{5}$ to $9.8 \times 10^{5}$. Image processing libraries of Mathematica Computational Software v8 were used to detect lens edges. The coordinates of detected points were fitted with higher order aspheric polynomials (as in Bahrami and Goncharov, 2012).

In addition to simulating reflected light a linear congruential generator algorithm (Park and Miller, 1988) was used to generate fine random fluctuations in the central lens to simulate scatter. The algorithm produces random vectors $\vec{\delta}$, where $0 \leq|\vec{\delta}| \leq 0.1 \mathrm{~mm}$ that add a minor fluctuation to the coordinates of the function $R(x, y, z)$. The fluctuations were higher in the axial regions and declined with distance away from the axis, simulating decreased scatter with distance from the centre of the lens based on morphological findings (Taylor et al., 1996; Al-Ghoul and Costello, 1997; Costello et al., 2007; Costello et al., 2008; Shestopalov and Bassnett, 2000).

\section{Results}

When the GRIN profile for a spherical radially symmetric structure is altered from a linear GRIN profile to a series of profiles, each an integral function of the previous one (Fig. 1A), the images of light reflection obtained from applying Fresnel's equations to the gradient index profiles appear as shown in Fig. 1B. These patterns are seen as long as the GRIN profile is relatively smooth with incremental changes that are negligible in comparison to the slope of the profile.

The bisecting dark line is visible in all patterns with only the linear profile showing a break in the line at the central point. This dark band is a result of the spacing between the iso-indicial contours. As collimated incident light travels from the left side of the lens to its right side (parallel to the optical axis $z$ ), the rays experience a slower gradient at the equatorial zone. This refractive index profile can be described as:

$n(r)=n_{c}+\left(n_{s}-n_{c}\right) r^{p}$

where $n_{c}$ is the refractive index of the centre of the lens, $n_{s}$ is the refractive index of its surface, $r$ is the normalised radial distance from the centre, and $p$ is an exponent which adjusts for the curvature of the profile.

As $p$ increases from 1 to 4 , the refractive index profile takes on a progressively flatter central region (Fig. $1 \mathrm{~A}$ ). When $p=1$, the lens shows a defined point of high reflectance at its centre with a relatively fine dark bow region emanating from this central point (Fig. 1B). With increasing value of the exponent $p$, this point is no longer seen and the bow becomes a straight bisecting line with the shadowing gradually decreasing towards the periphery. This gradual decrease occurs along a perpendicular to the dark bisecting line for $p=2$ and becomes more radially varying for higher values of $p$ (Fig. 1B). The central dark line continues to be visible for $p=3$ and $p=4$ and the light reflection is greatest in the peripheral regions; the variation in the amount of reflectance takes on an ovalshaped form (Fig. 1B).

Applying equation (1) to a lens model with well-defined isoindicial contours that follow a lenticular surface shape (Fig. 2A) gives a reflectance pattern shown in Fig. 2B. The value of $p=4$, $n_{C}=1.415$, and $n_{S}=1.37$ (Pierscionek, 2009; Bahrami and Goncharov, 2012). The oval-shaped darker region, which indicates minimal reflection, is evident and here the dark bisecting feature is not a straight line but is slightly curved (Fig. 2B). 
A
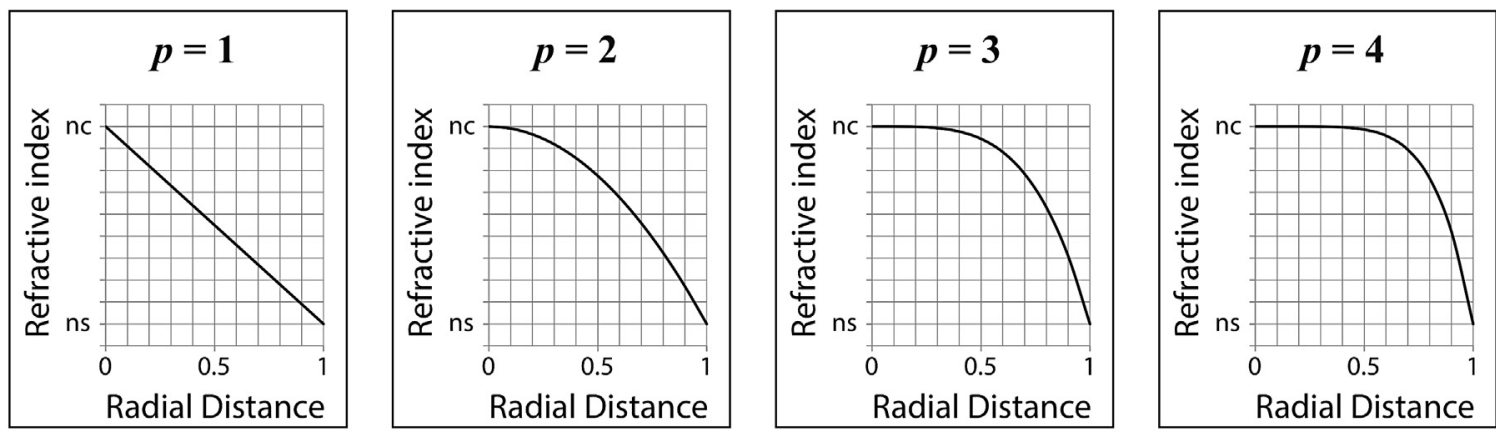

B

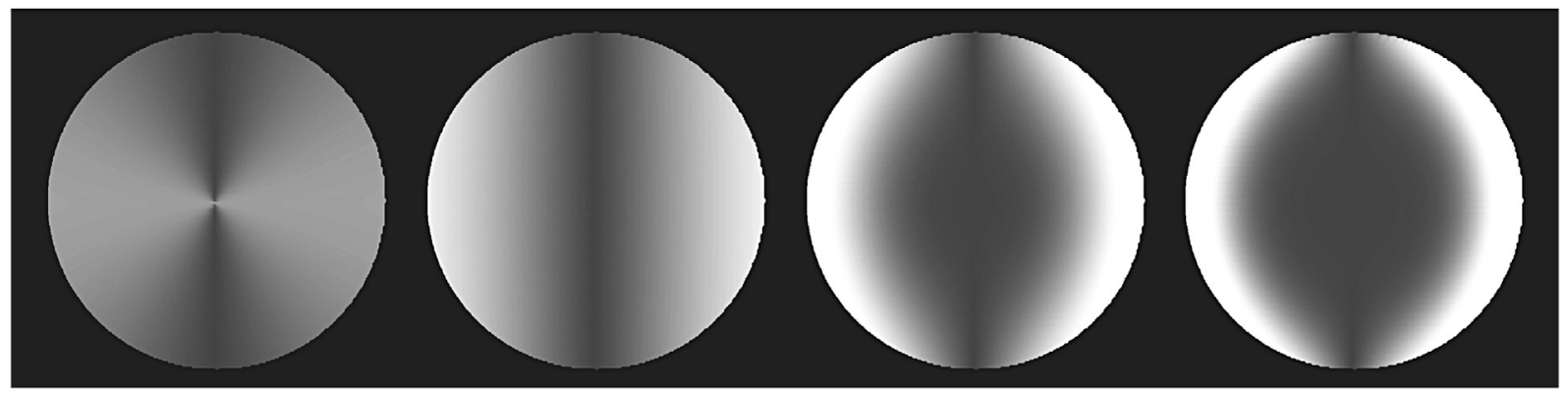

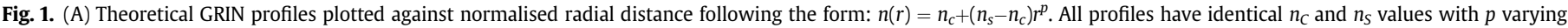
from 1 to 4; (B) Light reflection patterns calculated from central planes of spherical lenses with the respective profiles seen in (A).

The refractive index variations along the optic axes of three human lenses, aged 16, 35, and 48 years, are shown in Fig. 3. In all three lenses, the gradient is steep in the outer half of each lens and fluctuations are evident in the central plateau region (Fig. 3A). The profile shapes suggest a power law fit with $p>3$. Simulating the patterns of reflection from the axial refractive index profiles (by applying equation (1) as for the models in Figs. 1 and 2) and using the actual shapes of the lenses, gives the images seen in Fig. $3 \mathrm{~B}$.
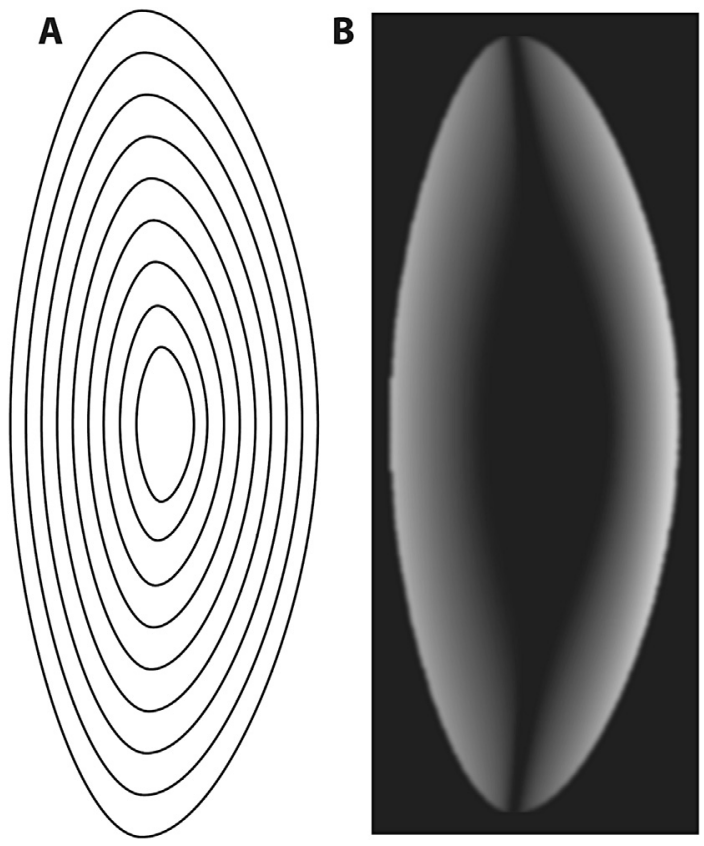

Fig. 2. (A) The structure of the iso-indicial contours for the lens model introduced by Bahrami and Goncharov (2012); (B) Light reflection patterns calculated in the sagittal plane.
The simulated reflectance patterns show bright and dark bands with the brightest regions in the periphery (Fig. $3 \mathrm{~B}$ ) where the refractive index profile is steepest (Fig. 3A). This would result in greater reflection of light (equation (1)). It is notable that the dark/ light zones are not all matched in the anterior and posterior regions eg a light band in the posterior section does not in all cases have a similar light band in the anterior of the lens. The dark bisecting band that runs approximately through the equatorial plane, becomes more curved as the lens shape becomes more asymmetric (Fig. 3B).

As measurements were conducted on in vitro samples, these lenses were free from the tensile forces that are mediated by the ciliary muscle in vivo. In order to make a comparison with a living lens, the simulated reflectance patterns for the 35 year old lens, were transposed to fit the shape of a living lens of comparable age (Rosales et al., 2006) (Fig. 4). The zones in the outer cortex appear brighter when the lens shape is transposed to the elongated, less accommodated state, as they become more 'compacted' and the contrast between light and dark bands increases.

The fibre cell organisation in the nucleus of the lens is less organised than in the cortical regions (Taylor et al., 1996; Al-Ghoul and Costello, 1997; Shestopalov and Bassnett, 2000) with structural features that can increase the proportion of scattered light (Jedziniak et al., 1975; Costello et al., 2007; Costello et al., 2008). This was simulated by superimposing random oscillations (Park and Miller, 1988) in the central regions and is depicted diagrammatically in Fig. 5A and B which show the entire lens and the enhanced central layers, respectively. The model used was continuous with an infinite number of isoindicial contours.

The additional simulation step to mimic the scattered light from central regions of the lens (Park and Miller, 1988) gives the image seen in Fig. 6A. This is compared with magnified Scheimpflug images in Fig. 6B and C (Rosales et al., 2006; Hermans et al., 2007 respectively). The dark bisecting curve, two central areas of scatter either side of this, a lighter zone in the cortical region and a thin dark zone in the sub-capsular region are seen in all images. 
A
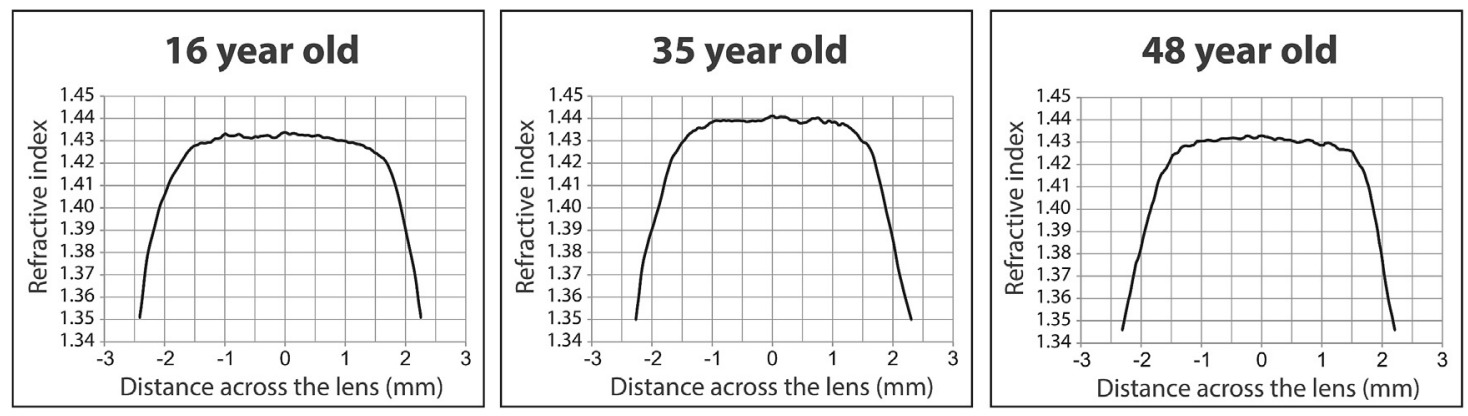

B

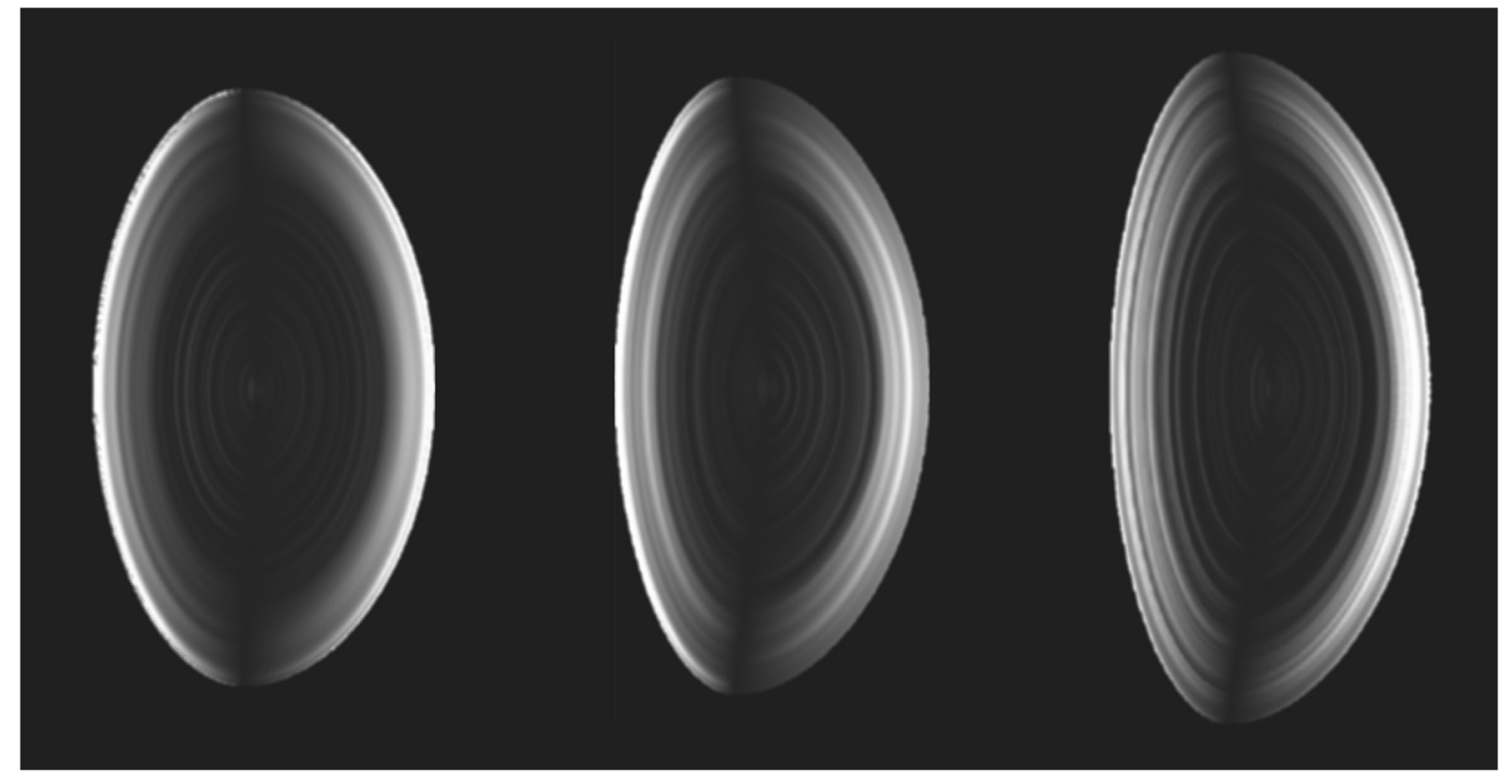

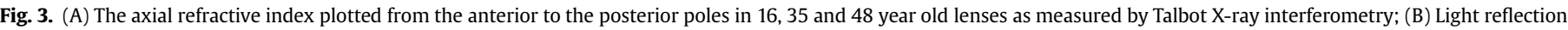
patterns calculated in sagittal planes of lenses in (A).

The clinical images have a slightly reduced contrast which may be because of intraocular sources of scatter that contribute to this reduction and the overall brightness of the simulation was matched to these for comparison. When the overall brightness of the image in Fig. 6A is increased, finer zones become more apparent (Fig. 7A) and can be compared to a pictorial representation of the zones of discontinuity which shows these features with greater clarity (Fig. 7B). It is possible to discern light and dark zones in the anterior capsular region, the wider band representing the cortex, the zones that define the adult and infantile nuclear regions and the segments that constitute the foetal and embryonic nuclei. Sizes of zones in the simulation and the pictorial representation vary. This may be attributed, to some extent, to artistic perspective.

\section{Discussion}

The design of non-uniform concentric, lamellar structures, created by new growth accruing over existing layers, is often found in Nature resulting in a chronological record of developmental and environmental changes. It is seen in pearls, tree rings and in the optics of the eye. The physical and earth scientists have long exploited growth modes and lamellar structures to learn about environmental changes; this has not been as prevalent in the eye. The eye lens is studied by medical and biomedical researchers for its physiological and pathological changes. The lens is an optical element and its structural features, their optical purpose and how changes in these features, in response to environmental and life style changes, alter optical function are questions that require further investigation. The answers will assist medicine in understanding ageing and in disease prevention and will provide necessary information to create improved designs for implant lenses.

In the human lens, the refractive index gradient has been fitted with a power law or a higher order polynomial function (Pierscionek and Chan, 1989; Pierscionek, 1990; Smith et al., 1991, 1992; Navarro et al., 2007; Kasthurirangan et al., 2008; Pierscionek, 2009). The subtle fluctuations in refractive index that have only been reported in the piscine lens (Kroger et al., 1994), have not been reported previously in the human lens. The hypothesis, that the zones seen in the live eye are created by subtle irregularities in the refractive index gradient (Pierscionek and Regini, 2012), is supported by the results of this work. The models have used experimental measurements of refractive index in human lenses to construct images of light reflection using Fresnel's equation. This quantitates the amount of reflected light based on refractive index and the changes in refractive index that occur when light traverses gradient index lenses. Simulations of fine random scatter were introduced in the central regions. The resulting image shows a strong resemblance to what is seen in the live human lens. In particular, features that appear consistently in 


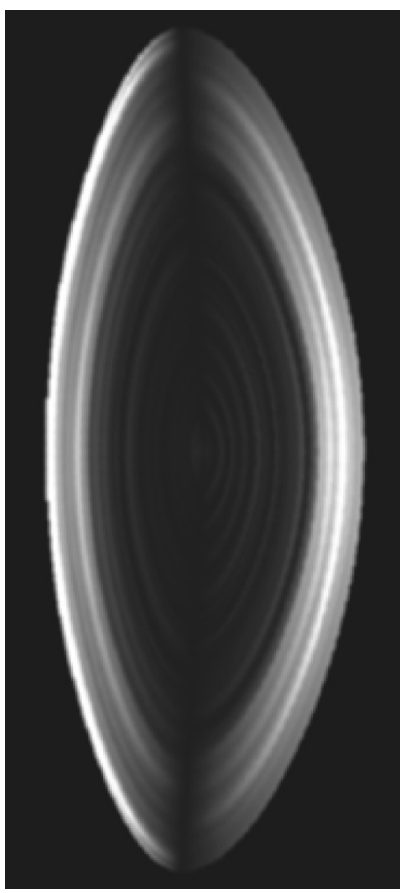

Fig. 4. Light reflection patterns for the 35 year old lens profile from Fig. 3(B) transposed to the geometry introduced in Fig. 2(A) to simulate the lens shape in vivo.

Scheimpflug images; a dark bisecting band through the plane of the equator, which is shown from this work to be an indication of a gradient index medium (Fig. 1), the brighter core region, and dark and light bands in both anterior and posterior regions of the lens, are seen in all images.

The simulation depicts many more rings than in the Scheimpflug image because in the living eye additional reflections that arise
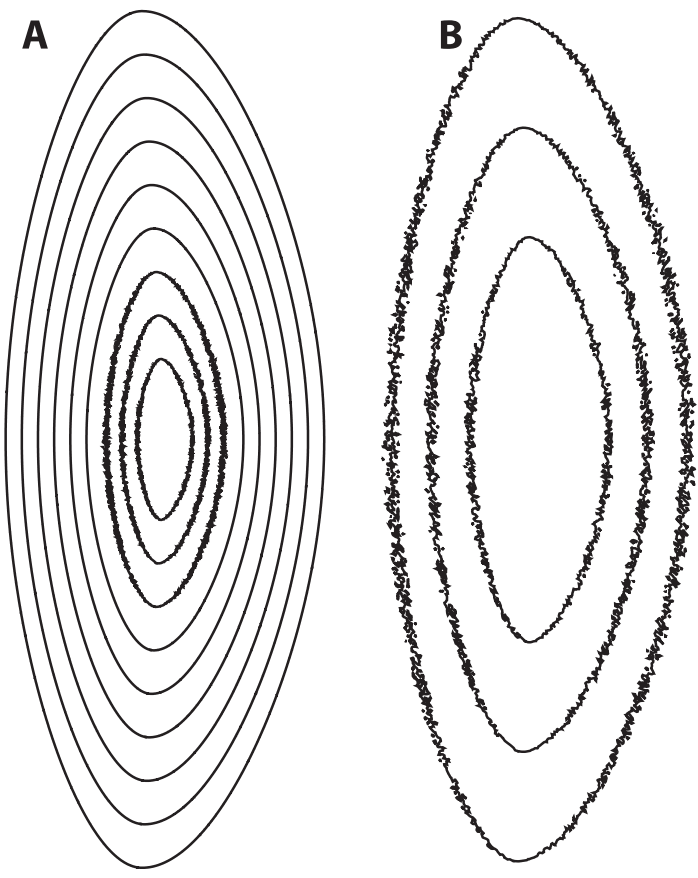

Fig. 5. (A) Diagrammatic representation of isoindicial contours in the whole lens with irregularities in the central regions; (B) Magnified view of the centre of the lens showing that fluctuations are highest in the axial region and decline with progression towards the equator.

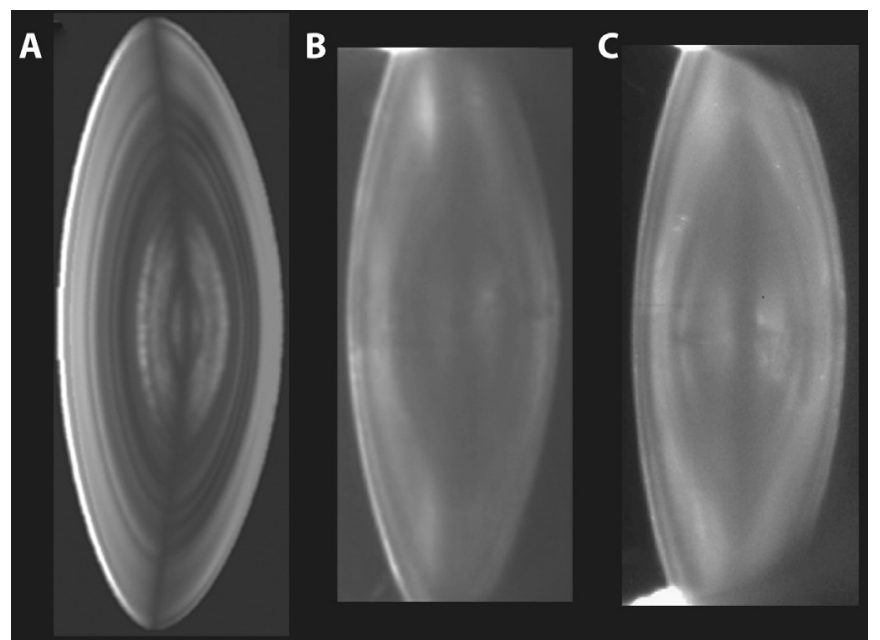

Fig. 6. (A) Simulation of reflected and scattered light for the GRIN profile of a 35 yearold lens compared to Scheimpflug images of (B) a young lens between the ages of 22 and 36 years (Rosales et al., 2006) and (C) a 28 year old lens (Hermans et al., 2007). The image in (B) was taken of the unaccommodated lens; the accommodative state of the image in (C) was not given but the shape suggests it is not accommodated or in a minimal state of accommodation.

from intraocular light scatter (van den Berg, 1997) can render the image less sharp than the simulation. In addition, the lenses from which refractive index measurements have been taken were postmortem lenses. The shape of the contours used in the model are regular and follow the surface shape; in the living lens there is a deviation from regularity in the central contours (Fig. 6B and C); (Rosales et al., 2006; Hermans et al., 2007) and the lens has been found to have rounder lamellae in the central regions with the outer lamellae taking on a more lenticular shape (Brown et al., 1988).

Simulating these variations in the inner contours would require a more complex mathematical model. Although it would refine the shapes of these contours, the findings that experimental measurements of refractive index fluctuations used in calculations of geometric scatter, produce zones of discontinuity, would be unchanged.

The zones of discontinuity have been described as isoindicial surfaces (Gullstrand, 1912, cited in Fagerholm et al., 1990) and well defined interfaces between regions of different refractive index (Mann, 1925; Huggert, 1948). Subsequent investigations on the subcapsular region of the anterior cortex in human lenses showed

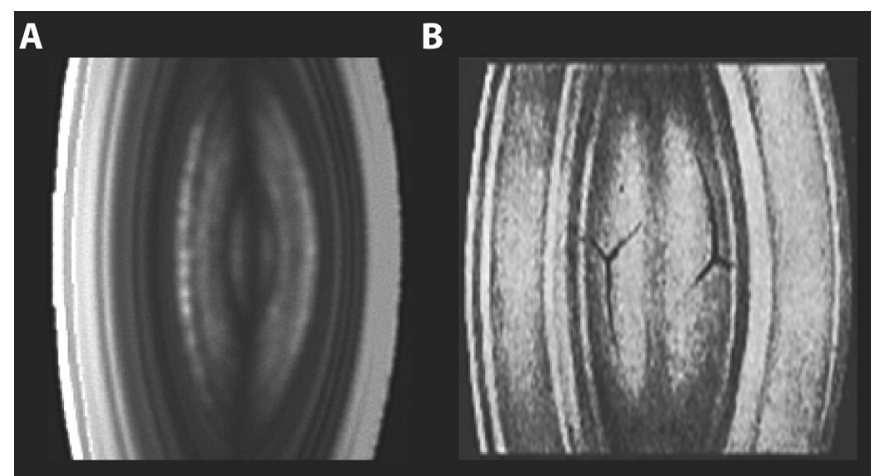

Fig. 7. (A) Simulation of reflected and scattered light for same GRIN profile of a 35 year-old lens as shown in Fig. 6(A) with overall brightness increased; (B) Anatomical drawing of the zones of discontinuity in a 40 year-old eye (Duke-Elder and Wybar, 1961). 
sharp shifts in protein concentration estimated to be between 0.003 and 0.009 change in refractive index. Protein concentration alone, however, cannot explain variations in light scatter along the optic axes of human lenses (Bettelheim and Paunovic, 1979; Yaroslavsky et al., 1994).

Around half of the light scattered within the eye has been ascribed to the membranes of lens fibre cells (Bettelheim and Paunovic, 1979; Hemenger, 1988). These membranes have a higher refractive index than the interior of the cell and in the eye lens this difference is most pronounced in the outer, cortical part of the lens (Michael et al., 2003). However, the fibre cell organisation is more regular in the cortex with greater irregularities and interdigitations between cell layers found in the inner one third of the lens (Taylor et al., 1996; Al-Ghoul and Costello, 1997). In addition, there is a predominance of structural features in the nucleus that contribute to light scatter. Jedziniak et al. (1975) found heavy or high molecular weight $(\mathrm{HMW})$ proteins $\left(>1.5 \times 10^{5} \mathrm{kDa}\right)$ in the soluble fractions of human lenses and that these can constitute around $10-15 \%$ of total soluble protein extract in normal 72 year old lenses. Aggregation of cytoplasmic proteins, particularly of $\gamma$ crystallin has been linked to increased light scatter (Yaroslavsky et al., 1994).

Changes in fibre cell membranes with age and cataract, such as breaks in membranes, enlargements of the extracellular space between membranes and protein-like deposits within the extracellular space (Costello et al., 2008) also scatter light. It is notable, that these changes were found in normal control lenses indicating that the process is age-related and may predispose to nuclear cataract rather than being specifically associated with the disease process (Costello et al., 2008). These changes are therefore physiological, as are the zones of discontinuity. Multilamellar bodies (MLBs), a term used by Gilliland et al. (2001) to describe spherical structures enclosed in multiple membrane layers, though occupying a very small volume fraction $\left(3 \times 10^{-7}\right.$ of the volume of a normal human lens) (Gilliland et al., 2001) occur in sufficient proportion to create significant light scatter (van den Berg and Spekreijse, 1999). These MLBs have been found in the foetal and embryonic nuclear regions (Gilliland et al., 2001) and it has been calculated that a small difference of 0.06 in refractive index between the MLB and the surrounding cytoplasm is sufficient to produce effective scatter (Costello et al., 2007). The relative contribution of the different structural elements that can produce light scatter is difficult to calculate as it is likely to vary between individual lenses as well as with age. In addition, the studies that have identified these features (HMW proteins, MLBs, cell membrane ruptures) have been conducted on in vitro tissue; how these appear in the living lens and how much they contribute to light scatter is not known. The work presented in this study did not consider the relative contributors to light scatter but rather simulated their collective effects recognising that these predominate in the nucleus.

When the brightness of the simulated image is increased (Fig. 7A), it is possible to see similarities with zones in classical anatomic drawings (Fig. 7B) (Duke-Elder and Wybar, 1961) and to identify bands that accord with the Oxford classification system (Sparrow et al., 1986). The outermost thin, bright band represents the capsule and epithelium and the adjacent dark band corresponds to $\mathrm{C} 1 \alpha$ which contains newly synthesised fibre cells (Michael and Bron, 2011). The bright band $C 1 \beta$ is also present in Fig. 7A but is less clear than $\mathrm{C} 1 \alpha$. This zone $(\mathrm{C} 1 \beta)$ is thought to represent a loss of transparency of the fibre cells that were previously in $\mathrm{C} 1 \alpha$ (Michael and Bron, 2011) and has been shown subsequently, from microscopic analysis, to contain a high number of cell interface inter-digitations and irregularities that could contribute to scatter (Costello et al., 2013). This region, termed the remodelling zone (RZ), contains cells that are undergoing structural rearrangement and has been postulated to be a region in which fibre cell differentiation changes abruptly (Lim et al., 2009). The outer part of the lens is where the refractive index gradient is steepest (Pierscionek and Chan, 1989; Pierscionek, 1990, 2009). Any fluctuations in the gradient in this region are more likely to indicate changes in cell growth and differentiation than fluctuations that appear in the central, nuclear region where refractive index is relatively flat and where scatter is caused by the more degenerative age-related processes.

The zones of discontinuity have been shown to change in number and position with age (Koretz et al., 1984, 1994). This is similar to what is seen in tree rings. It has recently been suggested that the zones of discontinuity may be indicative of changes in growth rate in response to developmental and/or environmental and lifestyle factors (Pierscionek and Regini, 2012). Zones of discontinuity have been observed in animals, aquatic (Dawson et al., 1992) and terrestrial (Koretz et al., 1988; Seeberger et al., 2004; Yuen et al., 2008) and these zones have yet to be probed as chronological features that could provide information about growth phases. The linking of these zones to refractive index offers the possibility of further modelling studies that can predict the biophysical changes that underlie the physiological and ultimately, the clinical picture.

More pertinent, is the question of whether the refractive index fluctuations, also have a functional use or whether they are entirely unintended for function and merely result from structural features associated with cells or from a growth related manifestation that indicates growth spurts and pauses, as is seen with tree growth rings. If there is a functional significance that can be attributed to these fluctuations, it must relate to the optics of the lens and the image quality this provides to the eye. The optics of the eye lens is superior to any intraocular implant thus far designed because a gradient index provides far better focussing ability and a significant reduction in aberrations than does a homogenous index lens. In addition, for a homogenous index lens to have the same refractive power as a gradient index lens would require either greater curvature and/or higher protein concentration rendering the human lens unable to accommodate by deformation. The presence of refractive index fluctuations, however, is not as well understood although it has been suggested, from a study on piscine lenses, that these features could reduce chromatic aberrations (Kroger et al., 1994). No implant has yet been made with an index gradient, let alone with fine fluctuations in the gradient. These fluctuations and the reflective zones they create in the image of the lens, vary with age (Koretz et al., 1984) and are likely to vary with individuals in accordance with growth and developmental phases. If they do indeed have an optical purpose, they are also likely to alter depending on the biometry of the eye, adjusting to parameters such as corneal refractive power and axial length of the eye.

Refractive index has been measured directly in a number of ways: ray tracing, fibre optic sensing and more recently with interferometric techniques (Pierscionek and Regini, 2012). It is a parameter that depends on wavelength and temperature and will alter if tissue dehydrates as can occur with invasive methods. It is therefore not surprising to find slightly varying values of refractive index when comparing different studies (Pierscionek and Regini, 2012). The method used in this work is an interferometric measurement and refractive index is calculated from density changes (Momose et al., 2003; Momose, 2005). It allows non-invasive measurement of the lens within the eyeball as do MRI methods (Kasthurirangan et al., 2008). The latter have the advantage that they can be conducted in vivo (Kasthurirangan et al., 2008) but are limited in that refractive index is calculated from protein concentrations derived from transverse relaxation times of water protons. This has relevance to soluble protein homogenates (Hills et al., 
1989) but takes no account of insoluble protein or the fact that water in the intact lens exist in free and bound states and that these produce differences in transverse relaxation times (Despa et al., 2008). These factors are particularly significant for any conclusions about age-related changes in refractive index given that with age there is an increase in both insoluble protein (Pirie, 1968; Satoh, 1972; Lahm et al., 1985) and free water (Lahm et al., 1985; Bettelheim et al., 2002; Heys et al., 2008). The X-ray Talbot interferometer takes around 900 scans/per lens and is a highly accurate method for direct measurement of tissue density changes enabling the detection of subtle fluctuations in density and refractive index. The measurements have produced results which, when used to model light reflectance and scatter, can simulate the zones of discontinuity. The findings have relevance to physiology and interpretation of growth related change and may lead to a better understanding of what is required in implant lenses in order to meet the high level of optical quality that the visual system demands.

\section{Acknowledgements}

This work is supported by Fight for Sight, grant no 1319/1320 and the authors gratefully acknowledge beamtime support at the Spring-8 synchrotron, Japan.

\section{References}

Al-Ghoul, K.J., Costello, M.J., 1997. Light microscopic variation of fiber cell size, shape and ordering in the equatorial plane of bovine and human lenses. Mol. Vis. 3 $2-8$.

Bahrami, M., Goncharov, A.V., 2012. Geometry-invariant gradient refractive index lens: analytical ray tracing. J. Biomed. Opt. 17, 0550011-0550019.

Bettelheim, F.A., Paunovic, M., 1979. Light scattering of normal human lens I. Application of random density and orientation fluctuation theory. Biophys. J. 26, 85-99.

Bettelheim, F.A., Lizak, M.J., Zigler Jr., J.S., 2002. Relaxographic studies of aging normal human lenses. Exp. Eye Res. 75, 695e702.

Born, M., Wolf, E., 1970. In: Principles of Optics, fourth ed. Pergamon Press, Oxford, pp. 36-51.

Brown, N.A.P., Sparrow, J.M., Bron, A.J., 1988. Central compaction in the process of lens growth as indicated by lamellar cataract. Br. J. Ophthalmol. 72, 538-544.

Costello, M.J., Johnsen, S., Gilliland, K.O., Freel, C.D., Fowler, W.C., 2007. Predicted light scattering from particles observed in human age-related nuclear cataracts using Mie scattering theory. Investig. Ophthalmol. Vis. Sci. 48, 303-312.

Costello, M.J., Johnsen, S., Metlapally, S., Gilliland, K.O., Ramamurthy, B. Krishna, P.V., Balasubramanian, D., 2008. Ultrastructural analysis of damage to nuclear fiber cell membranesin advanced age-related cataracts from India. Exp. Eye Res. 87, 147-158.

Costello, M.J., Mohamed, A., Gilliland, K.O., Fowler, W.C., Johnsen, S., 2013. Ultrastructural analysis of the human lens fiber cell remodelling zone and the initiation of cellular compaction. Exp. Eye Res. 116, 411-418.

Dawson, W.W., Hope, G.M., Dawson, J.C., Nachtigall, P.E., Schroeder, J.P., 1992 Cetacean lens zones of discontinuity - indices of health and development. Mar. Mamm. Sci. 8, 379-386.

Despa, F., Fernández, A., Scott, L.R., Berry, R.S., 2008. Hydration profiles of amyloidogenic molecular structures. J. Biol. Phys. 34, 577-590.

Duke-Elder, S., Wybar, K.C., 1961. System of ophthalmology. In: The Anatomy of the Visual System, vol. II. The CV Mosby Company, St Louis.

Fagerholm, P., Philipson, B.T., Lydahl, E., 1990. Subcapsular zones of discontinuity in the human lens. Ophthalmic Res. 22, 51-55.

Gilliland, K.O., Freel, C.D., Lane, C.W., Fowler, W.C., Costello, M.J., 2001. Multilamellar bodies as potential scattering particles in human age-related nuclear cataracts. Mol. Vis. 7, 120-130.

Goldmann, H., 1937. Studien uber den Alterskernstreifen der Linse. Arch. Augenheilk 110, 414-415.

Hemenger, R.P., 1988. Small-angle intraocular light scatter: a hypothesis concerning its source. J. Opt. Soc. Am. A 5, 577-582.

Hermans, E., Dubbelman, M., van der Heijde, R., Heethaar, R., 2007. The shape of the human lens nucleus with accommodation. J. Vis. 7, 1-10.

Heys, K.R., Friedrich, M.G., Truscott, R.J.W., 2008. Free and bound water in norma and cataractous human lenses. Investig. Ophthalmol. Vis. Sci. 49, 1991-1997.

Hills, B.P., Takacs, S.F., Belton, P.F., 1989. The effects of proteins on the proton NMR transverse relaxation times of water. I. Native bovine serum albumin. Mol. Phys. 67, 903-918.

Hoshino, M., Uesugi, K., Yagi, N., Mohri, S., Regini, J., Pierscionek, B., 2011. Optical properties of in situ eye lenses measured with X-Ray Talbot interferometry: a novel measure of growth processes. PLoS One 6, e25140.
Huggert, A., 1948. On the form of the isoindicial surfaces of the human crystalline lens. Acta Ophthalmol. (Copenh) (Suppl.) 30,1-126.

Jedziniak, J.A., Kinoshita, J.H., Yates, E.M., Benedek, G.B., 1975. The concentration and localization of heavy molecular weight aggregates in aging normal and cataractous human lenses. Exp. Eye Res. 20, 367-369.

Kasthurirangan, S., Markwell, E.L., Atchison, D.A., Pope, J.M., 2008. In vivo study of changes in refractive index distribution in the human crystalline lens with age and accommodation. Investig. Ophthalmol. Vis. Sci. 49, 2531-2540.

Koretz, J.F., Cook, C.A., Kuszak, J.R., 1994. The zones of discontinuity in the human lens: development and distribution with age. Vis. Res. 34, 2955-2962.

Koretz, J.F., Handelman, G.H., Brown, N.P., 1984. Analysis of human crystalline lens curvature as a function of accommodative state and age. Vis. Res. 24, 1141-1151.

Koretz, J.F., Bertasso, A.M., Neider, M.W., Kaufman, P.L., 1988. Slit-lamp studies of the rhesus monkey eye: III. The zones of discontinuity. Exp. Eye Res. 46, $871-880$.

Kroger, R.H.H., Campbell, M.C.W., Munger, R., Fernald, R.D., 1994. Refractive index distribution and spherical aberration in the crystalline lens of the African Cichlid fish Haplochromis burtoni. Vis. Res. 34, 1815-1822.

Lahm, D., Lee, L.K., Bettelheim, F.A., 1985. Age dependence of freezable and nonfreezable water content of normal human lenses. Investig. Ophthalmol. Vis. Sci. 26, 1162-1165.

Lim, J.C., Walker, K.L., Sherwin, T., Schey, K.L., Donaldson, P.J., 2009. Confocal microscopy reveals zones of membrane remodeling in the outer cortex of the human lens. Investig. Ophthalmol. Vis. Sci. 50, 4304-4310.

Mann, I.C., 1925. Notes on the anatomy of the living eye, as revealed by the Gullstrand Slitlamp. J. Anat. 59, 155-165.

Michael, R., van Marle, J., Vrensen, G.F., van den Berg, T.J., 2003. Changes in the refractive index of lens fibre membranes during maturation-impact on lens transparency. Exp. Eye Res. 77, 93-99.

Michael, R., Bron, A.J., 2011. The ageing lens and cataract: a model of normal and pathological ageing. Philos. Trans. R. Soc. B 366, 1278-1292.

Momose, A., 2005. Recent advances in X-ray phase imaging. Jpn. J. Appl. Phys. 44, 6355-6367.

Momose, A., Kawamoto, S., Koyama, I., Hamaishi, Y., Takai, K., Suzuki, Y., 2003. Demonstration of X-ray Talbot interferometry. Jpn. J. Appl. Phys. 42, L866-L868.

Navarro, R., Palos, F., González, L., 2007. Adaptive model of the gradient index of the human lens. I. Formulation and model of aging ex vivo lenses. J. Opt. Soc. Am. A 24, 2175-2185.

Park, S.K., Miller, K.W., 1988. Random number generators: good ones are hard to find. Commun. ACM 31, 1192-1201.

Pierscionek, B.K., Regini, J.W., 2012. The gradient index lens of the eye: an optobiological synchrony. Prog. Retin. Eye Res. 31, 332-349.

Pierscionek, B.K., 1990. Presbyopia - effect of refractive index. Clin. Exp. Optom. 73, 23-30.

Pierscionek, B.K., Chan, D.Y.C., 1989. The refractive index gradient of the human lens. Optom. Vis. Sci. 66, 822-829.

Pierscionek, B.K., 2009. Gradient index of refraction (GRIN) profiling of the eye lens. Chapter 19. In: Bass, M., Enoch, J., Mahajan, V.N., DeCustatis, Lakshminarayanan, V. (Eds.), Handbook of OpticsMcGraw-Hill, New York, 19.1-19.18.

Pirie, A., 1968. Colour and solubility of the proteins of human cataracts. Investig. Ophthalmol. 7, 634-650.

Rosales, P., Dubbelman, M., Marcos, S., Van der Heijde, R., 2006. Crystalline lens radii of curvature from Purkinje and Scheimpflug imaging. J. Vis. 6, 1057-1067.

Satoh, K., 1972. Studies on the structural proteins of the human lens. Exp. Eye Res. $14,53-57$.

Seeberger, T.M., Matsumoto, Y., Alizadeh, A., Fitzgerald, P.G., Clark, J.I., 2004. Digital image capture and quantification of subtle lens opacities in rodents. J. Biomed. Opt. 9, 116-120.

Shestopalov, V.I., Bassnett, S., 2000. Three dimensional organisation of primary lens fiber cells. Investig. Ophthalmol. Vis. Sci. 41, 859-863.

Smith, G., Pierscionek, B.K., Atchison, D.A., 1991. The optical modelling of the human lens. Ophthalmic Physiol. Opt. 11, 359-369.

Smith, G., Atchison, D.A., Pierscionek, B.K., 1992. Modeling the power of the aging human eye. J. Opt. Soc. Am. A 9, 2111-2117.

Sparrow, J.M., Bron, A.J., Brown, N.A.P., Ayliffe, W., Hill, A.R., 1986. The Oxford clinical cataract classification and grading system. Int. Ophthalmol. 9, 207-225.

Taylor, V.L., Al-Ghoul, K.J., Lane, C.W., Davis, V.A., Kuszak, J.R., Costello, M.J., 1996. Morphology of the normal human lens. Investig. Ophthalmol. Vis. Sci. 37, 1396-1410.

van den Berg, T.J., 1997. Light scattering by donor lenses as a function of depth and wavelength. Investig. Ophthalmol. Vis. Sci. 38, 1321-1332.

van den Berg, T.J., Spekreijse, H., 1999. Light scattering model for donor lenses as a function of depth. Vis. Res. 39, 1437-1445.

Vogt, A., 1919. Das farbenschillendeshinteren linsenbildes. Klin.Mbl.Augen-helik 62, 582.

Vogt, A., 1931. In: Lehrbuch und atlas der spaltlampenmikroskopie des auges, Zweiter Teil. Springer, Berlin.

Yaroslavsky, I.V., Yaroslavsky, A.N., Otto, C., Puppels, G.J., Vrensen, G.F.J.M., Duindam, H., Greve, J., 1994. Combined elastic and Raman light scattering of human eye lenses. Exp. Eye Res. 59, 393-400.

Yuen, J., Li, Y., Shapiro, L.G., Clark, J.I., Arnett, E., Sage, E.H., Brinkley, J.F., 2008. Automated, computerized, feature-based phenotype analysis of slit lamp images of the mouse lens. Exp. Eye Res. 86, 562-575. 